\title{
High Risk Myelodyplastic Syndrome with Good Risk Cytogenetics and Somatic Oncogenic Driver Mutation
}

\author{
Samra Waheed*, Saba Shahid, Jawwad Hasan, Shariq Ahmed, Tahir S. Shmasi \\ National Institute of Blood Disease \& Bone Marrow Transplantation, Karachi, Pakistan.
}

\begin{abstract}
Recurrent cytogenetic abnormalities are manifested in approximately fifty percent cases of Myelodysplastic Syndromes (MDS) found as result of genomic instability verifying the presence of oncogenic genetic mutations. Over the years the molecular diagnosis of MDS, has emphasized the relevance of the molecular pathogenesis of this entity by utilizing the refined technology of next generation sequencing. We herein report a case of Myelodysplastic Syndrome with Excessive Blast 2(MDS EB 2) with isolated deletion 5q and the presence of oncogenic somatic driver mutation ASXL-1 elucidated through next generation sequencing. The reports pertaining the association of MDS with deletion $5 \mathrm{q}$ and ASXL 1 are relatively exiguous. This case report points towards the diagnostic and prognostic significance of somatic driver mutations, even in patients exhibiting good risk cytogenetics. This will assist in offering better risk adapted therapies in Myelodysplastic syndrome patients.
\end{abstract}

Keywords: Myelodysplastic syndrome, Next Generation Sequencing, Deletion 5q, ASXL-1 mutation, Acute myeloid leukemia, Azacitidine, Oncogenic mutations.

\section{INTRODUCTION}

The myelodysplastic syndrome (MDS) is a heterogeneous collection of hematological disorders manifested mostly by defective hematopoiesis resulting in varied cytopenias and an increased risk of converting into acute myeloid leukemia (AML) [1]. The pathogenesis that causes these pre-leukemic disorders is unclear yet, but immune deregulation along with abnormal microenvironment and augmentation of genetic alterations may all have important roles [2]. Recurrent cytogenetic abnormalities are demonstrated in approximately fifty percent cases of MDS and are an important tool of establishing clonality and defining prognosis [3]. Among karyotypic abnormalities, partial or complete deletion of the long arm of chromosome $5[\operatorname{del}(5 q)]$ is the most frequent cytogenetic abnormality found in MDS patients, occurring in $10-15 \%$ of all newly diagnosed MDS patients and in up to $30 \%$ of cases with additional abnormal cytogenetic abnormality [4]. The prognosis of MDS cases with $\operatorname{del}(5 q)$ worsens with additional cytogenetic changes and increase in blast percentages. Over the years the molecular basis of MDS has remained elusive but major breakthroughs have been made recently in elucidating the molecular pathogenesis of this entity by employing the sophisticated technology of next generation sequencing [5]. Some of these determined mutations play the role of somatic foundation mutations and others are considered to be subclonal. Mutations in ASXL1 (additional sex combs 1) have been identified in MDS [6] as well as other myeloid malignancies, including acute myeloid leukemia (AML) [7], chronic myelomonocytic leukemia (CMML) [8] and myeloproliferative neoplasms. The function

*Address correspondence to this author at the National Institute of Blood Disease \& Bone Marrow Transplantation, Karachi, Pakistan.

E-mail: samraw2010@gmail.com of ASXL1 protein is not fully defined, but it is proposed that it may be involved in DNA and/or histone modification [9]. ASXL1 mutations almost all are disclosed in exon 12 of the gene and are believed to lead to the truncation of the plant homeodomain 10 at the $\mathrm{C}$-terminus of the protein, which is associated in chromatin modification. ASXL1 mutation is found in a significant proportion (11-18.5\%) of WHO defined MDS patients [10]. Weherein report a patient of myelodysplastic syndrome with excessive blast 2 (MDS- EB2) along with isolated del5q and presence of ASXL-1 mutation.

\section{CASE REPORT}

A 62 years old male with no known comorbid presented to us with concerns of weakness, vertigo and shortness of breath on exertion. On examination, pallor was the only positive sign, rest of the general and systemic examination was normal. There was no history of blood transfusion.

He was referred to National Institute of Blood Disease and Bone Marrow Transplant for the evaluation of macrocytic anemia after receiving appropriate doses of hematinics for 03 months. His serum Vitamin B12 and Serum and RBC folate levels were in normal limits.His complete blood count showed $\mathrm{Hb}$ of $6.8 \mathrm{~g} / \mathrm{dl}$ with $\mathrm{MCV}$ of $114 \mathrm{fl}, \mathrm{MCH}$ of $32 \mathrm{pg}$ and $\mathrm{MCHC} 33 \mathrm{~g} / \mathrm{dl}$. WBC count was $10.5 \times 10^{9} / \mathrm{L}$ with ANC of $1.8 \times 10^{9} / \mathrm{L}$ and platelet count of $109 \times 10^{9} / \mathrm{L}$. Peripheral smear revealed bicytopenia, macrocytosisalong with dysplastic, hypolobated and hypogranular neutrophils and $4 \%$ blast cells as shown in Fig. (1). Biochemical studies turned out to be normal and viral serology was negative, Ultrasound abdomen had no abnormal findings. 
Bone marrow aspirate along with trephine biopsy was performed, revealing hyperplastic bone marrow with cellularity of $80 \%$ with significant dysplasia and in myeloid and megakaryocytic lineage, (Hypogranular and hypolobated myeloid precursors, multinuclear megakaryocytes) as shown in Fig. (2) and (3). Bone marrow aspirate showed 14\% blast cells (500 cell count). Iron stain showed grade II iron with no ringed sideroblasts and reticulin stain showed MF-0. CD 34 was applied on trephine biopsy and showed positivity in $15 \%$ of the cells (Fig. $\mathbf{4}$ and 5). On the basis of above mentioned findings, diagnosis of Myelodysplastic syndrome with excess blast 2 was established. Bone marrow karyotyping showed 46, $\mathrm{XY}$, del [5][ q33.1] in 20 metaphases.

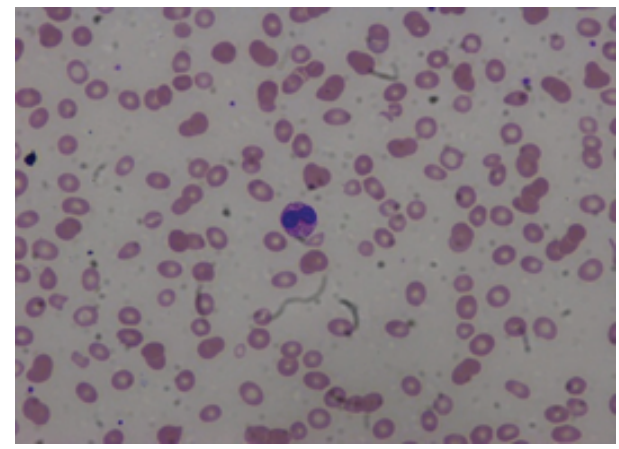

Fig. (1). Peripheral Smear Showing Dysplastic Bilobed Neutrophils.

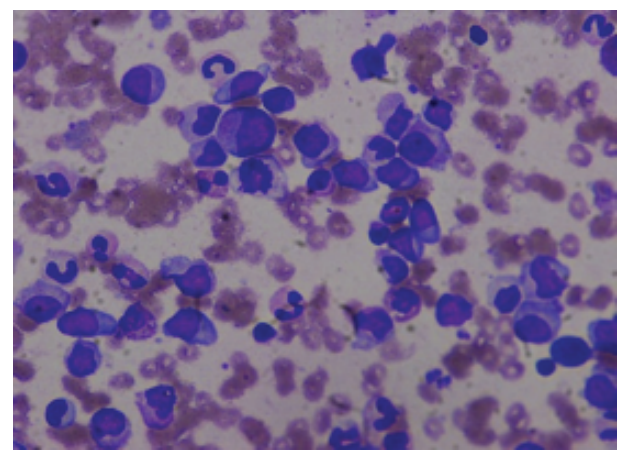

Fig. (2). Bone Marrow Aspirate Showing Hypogranular Myeloid Precursors and Myeloid Blast Cells.

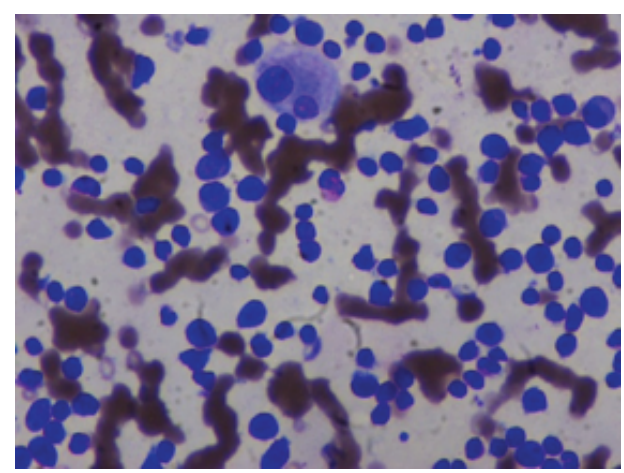

Fig. (3). Bone Marrow Aspirate Showing Dysplastic Multinuclear Megakaryocytes.

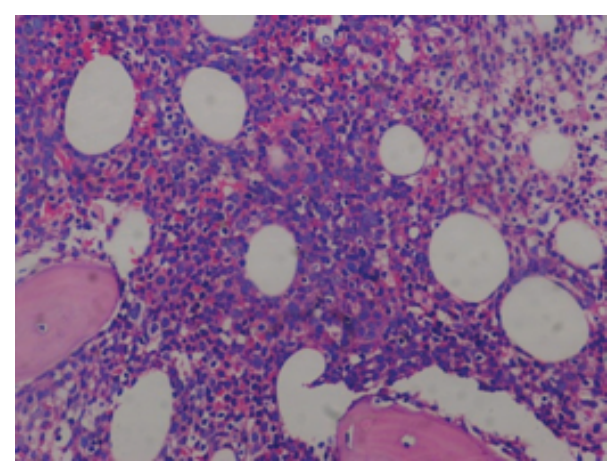

Fig. (4). Bone Marrow Trephine Showing Interstitial Infiltration By Blast Cells.

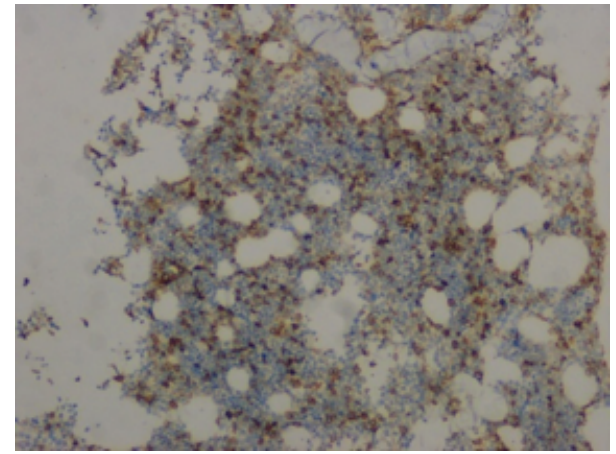

Fig. (5). CD34 Immunohistochemistry on Bone Marrow Trephine.

These investigations were then followed by next generation sequencing to sort out presence of any somatic driver mutation.

This case report was approved by the Institutional Review Board (ERC/IRB) and conformed to the tenets of the Declaration of Helsinki. Written informed consent was obtained from the patient. The myeloid sequencing panel of 54 genes (complete coding exons of 15 genes and exonic hotspots of 39 genes) was sequenced. The panel total coverage was $141 \mathrm{~kb}$ in genomic sequence. TruSight myeloid sequencing (Illumina, CA) libraries were prepared and run was performed on a MiSeq (Illumina) genome sequencer. The generated data was analyzed by on-instrument software or TruSeqAmplicon $^{\circledR}$ and BaseSpace Apps $^{\circledR}$. The mutations identified as pathogenic were confirmed using the Sanger method according to the standard protocol $\left(\mathrm{BigDye}^{\circledR}\right.$ Terminator v3.1 Cycle Sequencing Kit, Applied Biosystems $^{\circledR}$ ).

Genomic analysis was performed using variant studio software v3 (Illumina, San Diego, CA, USA). Several databases, such as dbSNP, COSMIC, ClinVar, Varsome, Polyphen, SIFT and Ensemble, were used to find the status of variant and report pathogenic mutation. Overall 57 variants were identified, including 1 stop codon, 4 deletions, 4 insertions and 48 variants found as single nucleotide variants 
(SNVs). Of the SNVs identified, 12 were synonymous variants, and 9 were missense variants in the TET2, BCORL1, GATA2, KDM6A, TP53 and IKZF1 genes. The remaining 27 variants include 3 splice region variants, 2 downstream variants and 22 intronic variants with a MAF value of $>1 \%$. We identified nonsense variant c.2077C >T, p. Arg693Ter (rs373221034) in ASXL1 gene causing on Mutation Taster and damaging on FATHHMM-MKL. This mutation possibly prevent proper transcription into RNA suggests they represent somatic and not germline events.

The patient was classified as high risk with IPSS-R with a calculated score of 5.3. Such patients usually have overall survival of 1.6 years and $25 \%$ chance to transform to Acute Myeloid Leukemia. As per current recommendations, patient is receiving Azacitidine 150mg subcutaneously for a week, every month. Patient uneventfully completed two cycles of azacytidine without much response and transfused thrice during that period. Unfortunately since after the second cycle the patient was lost to follow up and no further information could be obtained as per the clinical status of the patient and progression of the disease.

\section{DISCUSSION}

According to the World Health Organization (WHO) 2016 classification, the only cytogenetic abnormality, representing a MDS subtype is the deletion of the long arm of chromosome 5 (del (5q)), conferring the diagnosis of MDS with isolated del(5q) [11]. Globally, less than 50\% of MDS patients display an aberrant karyotype, of which del( $5 q)$ is the most common cytogenetic abnormality displaying in 10 to $20 \%$ of MDS patients. In about $55 \%$ of patients with del(5q) this aberration appears as a sole abnormality, in $17 \%$ it presents with one additional aberration, while in another $28 \% \operatorname{del}(5 \mathrm{q})$ appears with two or more additional cytogenetic lesions [12]. Patients who have MDS with isolated del(5q) show a lower risk for progression to acute myeloid leukemia (AML), however recent advances in molecular genetics have rotated the clinical spectrum of the disease.Current literature implied approximately 50-60 genes as myelodysplasia driver genes. Moreover, Genotype - phenotype association has also been illustrated for various driver mutations [13]. Many genetic mutations have been identified by next generation sequencing in MDS patients possessing del(5q), changing the perception of good prognosis in such patients. One such mutation ASXL 1 has been indentifiedin our patient [14].

In MDS patients, ASXL1 mutation is statistically closely correlated with trisomy 8 and somatic genetic mutations including RUNX1, EZH2, IDH, NRAS, JAK2, SETBP1 and SRSF2 [15].

ASXL1 belongs to the intensifieroftrithorax and polycomb (ETP) genes that can both activateorrepressHoxgenes. Itcontainsseveral nuclear receptor binding motifs and acarboxy-ter- minal plant homeodomain (PHD) that is anticipated to be trimmed by most ASXL1 mutations found in patients [16]. ASXL1 can act as a transcriptional activator or co repressor of retinoic acid receptor (RAR) activity, confiding on cellular conditions [17]. ASXL 1 can either bediagnosticated as heterozygousframeshift mutations or the heterozygousmissensemutations. Frameshift mutations are affiliatedwith poorer prognosis, decreased overall survival and lesssens response to the therapy [18].

Data representing the presence of del5q along with ASXL 1 is sparse as both have reciprocal relationship with respect to prognosis. One such study, reported the presence of ASXL1 mutation with del $5 \mathrm{q}$ in $6 \%$ of the patients [19]. Most of the patients were transformed to AML, verifying the lethal nature of the mutation. Thus, ASXL1 has been demonstrated as an independent predictor of poor outcome [7, 18].

Lenalidomide has been associated with better overall survival in patients with isolated del 5q, one study has in sighted the fact that use of Lenalidomide in high risk patients with identified genetic mutations exhibiting poorer prognosis, Lenalidomide has been associated with decreased overall survival, poorer cytogenetic response and increased risk of transformation of disease [20].

This case report guides towards the diagnostic and prognostic importance of somatic driver mutations, even in patients exhibiting good risk cytogenetics. This will aid in offering better risk adapted therapies in MDS patients.

\section{FUNDING}

No fundings have been associated with the article.

\section{CONFLICT OF INTEREST}

Declared none.

\section{ACKNOWLEDGEMENTS}

We acknowledge all our fellow colleagues.

\section{REFERENCES}

[1] Heaney M, Golde D. Myelodysplasia. New Engl J Med 1999; 340(21): 1649-60. DOI: 10.1056/NEJM199905273402107

[2] Haase D, Germing U, Schanz J, et al. New insights into the prognostic impact of the karyotype in MDS and correlation with subtypes: evidence from a core dataset of 2124 patients. Blood 2007; 110(13): 4385-95.

DOI: 10.1182/blood-2007-03-082404

[3] Solè F, Lu-o E, Sanzo C, et al. Identification of novel cytogenetic markers with prognostic significance in a series of 968 patients with primary myelodysplastic syndromes. Haematologica 2005; 90: 1168-78. 
[4] Cazzola M, Della Porta M, Malcovati L. The genetic basis of myelodysplasia and its clinical relevance. Blood 2013; 122(25): 4021-34. DOI: 10.1182/blood-2013-09-381665

[5] Kulasekararaj AG, Mohamedali AM, Mufti GJ. Recent advances in understanding the molecular pathogenesis of myelodysplastic syndromes. Br J Haematol 2013; 162(5): 587-605. DOI: $10.1111 /$ bjh. 12435

[6] Rocquain J, Carbuccia N, Trouplin V, et al. Combined mutations of ASXL1, CBL, FLT3, IDH1, IDH2, JAK2, KRAS, NPM1, NRAS, RUNX1, TET2 and WT1 genes in myelodysplastic syndromes and acute myeloid leukemias. BMC Cancer 2010; 10: 401. DOI: 10.1186/1471-2407-10-401

[7] Chou WC, Huang HH, Hou HA, et al. Distinct clinical and biological features of de novo acute myeloid leukemia with additional sex comb-like 1 (ASXL1) mutations. Blood 2010; 116: 4086-94. DOI: 10.1182/blood-2010-05-283291

[8] Gelsi-Boyer V, Trouplin V, Adelaide J, et al. Mutations of polycomb-associated gene ASXL1 in myelodysplastic syndromes and chronic myelomonocyticleukaemia. $\mathrm{Br} \mathrm{J}$ Haematol 2009; 145: 788-800.

DOI: $10.1111 / \mathrm{j} .1365-2141.2009 .07697 . x$

[9] Pena PV, Davrazou F, Shi X, et al. Molecular mechanism of histone $\mathrm{H} 3 \mathrm{~K} 4 \mathrm{me} 3$ recognition by plant homeodomain of ING2. Nature 2006; 442: 100-3. DOI: 10.1038/nature04814

[10] Boultwood J, Perry J, Pellagatti A, et al. Frequentmutationofthe polycomb-associatedgeneASXL1in the myelodysplastic syndromes and in acute myeloid leukemia. Leukemia 2010; 24: 1062-5. DOI: 10.1038/leu.2010.20

[11] Bernasconi P, Klersy C, Boni M, et al. World Health Organization classification in combination with cytogenetic markers improves the prognostic stratification of patients with de novo primary myelodysplastic syndromes. Br J Haematol 2007; 137(3): 193-205. DOI: 10.1111/j.1365-2141.2007.06537.x

[12] Mallo M, Cervera J, Schanz J, et al. Impact of adjunct cytogenetic abnormalities for prognostic stratification in patients with myelodysplastic syndrome and deletion $5 \mathrm{q}$. Leukemia
2011; 25(1): 110-20. DOI: 10.1038/leu.2010.231

[13] Walter MJ, Shen D, Ding L, et al. Clonal architecture of secondary acute myeloid leukemia. New Engl J Med 2012; 366(12): 1090-8. DOI: 10.1056/NEJMoa1106968

[14] Scharenberg C, Giai V, Pellagatti A, et al. Progression in patients with low- and intermediate-1-risk del(5q) myelodysplastic syndromes is predicted by a limited subset of mutations. Haematologica 2017; 102: 498-508.

DOI: $10.3324 /$ haematol.2016.152025

[15] Chen T, Hou H, Chou W, et al. Dynamics of ASXL1 mutation and other associated genetic alterations during disease progression in patients with primary myelodysplastic syndrome. Blood Cancer J 2014; 4(1): e177-e7. DOI: 10.1038/bcj.2013.74

[16] Fisher CL, Pineault N, Brookes C, et al. Lossof-function additional sex combs like 1 mutations disrupt hematopoiesis but do not cause severe myelodysplasia or leukemia. Blood 2010; 115: 38-46. DOI: 10.1182/blood-2009-07-230698

[17] Cho YS, Kim EJ, Park UH, et al. Additional sex comb-like 1 (ASXL1), in cooperation with SRC-1, acts as a ligand-dependent coactivator for retinoic acid receptor. J Biol Chem 2006; 281: 17588-98. DOI: 10.1074/jbc.M512616200

[18] Thol F, Friesen I, Damm F, et al. Prognostic significance of ASXL1 mutations in patients with myelodysplastic syndromes. J Clin Oncol 2011; 29(18): 2499-506.

DOI: $10.1200 / J C O .2010 .33 .4938$

[19] Meggendorfer M, Haferlach C, Kern W, Haferlach T. Molecular analysis of myelodysplastic syndrome with isolated deletion of the long arm of chromosome 5 reveals a specific spectrum of molecular mutations with prognostic impact: A study on 123 patients and 27 genes. Haematologica 2017; 102(9): 1502-10. DOI: 10.3324/haematol.2017.166173

[20] Mallo M, Del Rey M, Ibanez M, et al. Response to lenalidomide in myelodysplastic syndromes with del $(5 q)$ : Influence of cytogenetics and mutations. Br J Haematol 2013; 162: 74-86. DOI: $10.1111 /$ bjh. 12354 